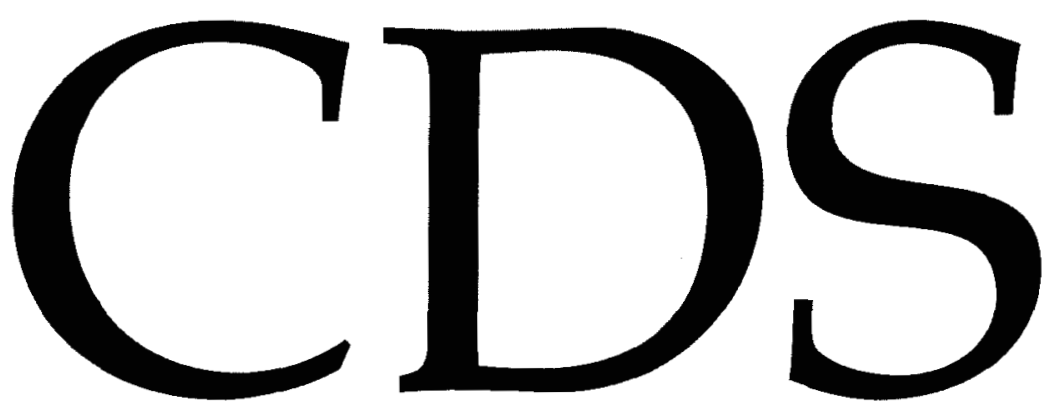

TECHNICAL MEMORANDUM NO. CIT-CDS 93-007

May 6, 1993

"Anti-Windup Design for Internal Model Control" Alex Zheng, Mayuresh V. Kothare and Manfred Morari

Control and Dynamical Systems California Institute of Technology Pasadena, CA 91125 


\title{
Anti-Windup Design for Internal Model Control
}

\author{
Alex Zheng \\ Mayuresh V. Kothare \\ Manfred Morari* \\ Chemical Engineering, 210-41 \\ California Institute of Technology \\ Pasadena, CA 91125
}

May 6, 1993

CIT-CDS Technical Memo

\begin{abstract}
This paper considers linear control design for systems with input magnitude saturation. A general anti-windup scheme which optimizes nonlinear performance, applicable to MIMO systems, is developed. Several examples, including an ill-conditioned plant, show that the scheme provides graceful degradation of performance. The attractive features of this scheme are its simplicity and effectiveness.
\end{abstract}

\section{Introduction}

Of special interest and common occurrence are systems having control input saturation nonlinearities but which are otherwise linear. Windup problems were originally encountered when using PI/PID controllers for controlling such systems. However, it was recognized later that integrator windup is only a special case of a more general problem. As pointed out by Doyle et al $[6]$, any controller with relatively slow or unstable modes will experience windup problems if there are actuator constraints. Windup is then interpreted as an inconsistency between the plant input and the states of the controller when the control signal saturates. The "conditioning technique" as an anti-windup technique was originally formulated by Hanus et al $[8,9]$ as an extension of the back calculation method of Fertik and Ross [7] to a general class of controllers. Åström and Wittenmark $[2,1]$ proposed that an observer be introduced into the system to estimate the states of the controller in the face of constraints and hence restore consistency between the saturated control signal and the controller states. This observer-based approach represented a significant generalization of the existing anti-windup schemes. Walgama and Sternby [11] have clearly exposed this inherent observer property in a large number of anti-windup schemes. Campo and Morari [3] have derived the Hanus conditioned controller independently as a special case of the observer-based approach.

The Internal Model Control (IMC) structure [10] (see Figure 1) was never intended to be an anti-windup scheme. Although stability of $P$ and $Q$ would guarantee global stability, provided that there is no plantmodel mismatch, the performance suffers when there are actuator constraints. This is because the controller

\footnotetext{
"To whom all correspondence should be addressed: phone (818)356-4186, fax (818)568-8743, e-mail: MM@IMC.CALTECH.EDU.
} 


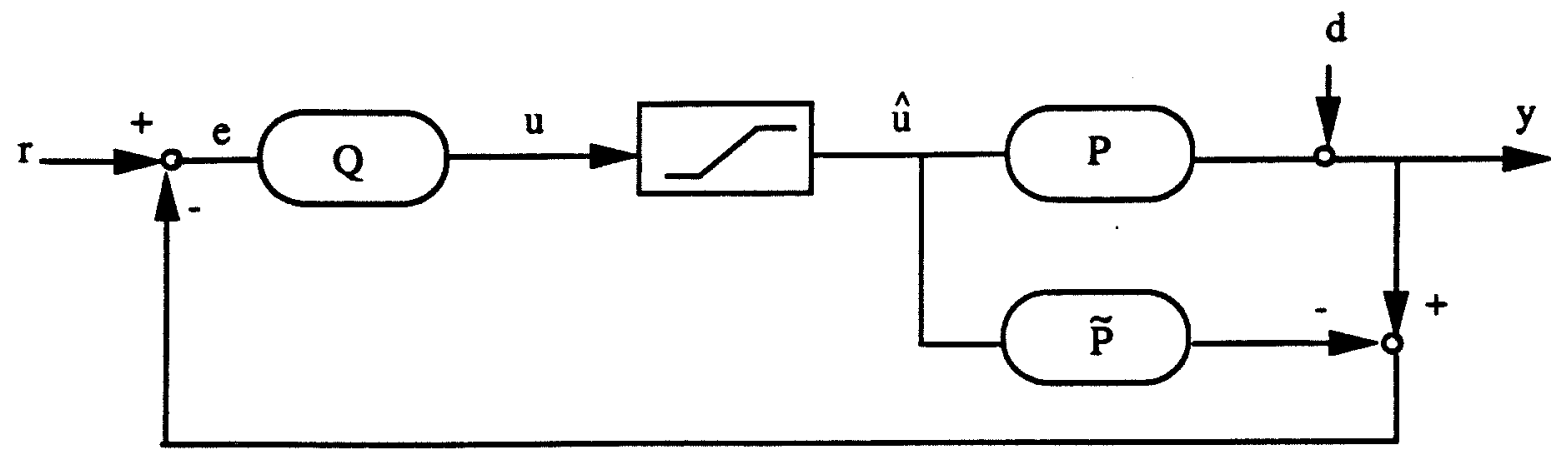

Figure 1: The IMC interconnection

$(Q)$ is entirely unaware of the effect of its action. In particular, it does not know if and when the manipulated variable $(u)$ saturates. This effect is most pronounced when the IMC controller has fast dynamics which are chopped off by the saturation. Unless the IMC controller is designed to optimize nonlinear performance, it will not give satisfactory performance for the saturating system. The focus of this paper is to identify this nonlinear performance.

Assumptions and Notations We will assume that the plant is a linear time invariant and stable square system with $n$ inputs and $n$ outputs. For simplicity, we will use the same symbol to denote both the transfer function and the corresponding impulse response model. The meaning should be clear from context. $P$, $\tilde{P}$, and $Q$ denote the plant, the model of the plant, and the IMC controller, respectively. They are $n$ by $n$ transfer matrices. For $u \in \Re^{n}, \operatorname{sat}(u)=\left\{\begin{array}{c}\operatorname{sat}\left(u_{1}\right) \\ \vdots \\ \operatorname{sat}\left(u_{n}\right)\end{array}\right.$, where $\operatorname{sat}\left(u_{i}\right)=\left\{\begin{array}{ll}u_{i}^{\max } & u_{i}>u_{i}^{\max } \\ u_{i} & u_{i}^{\min } \leq u_{i} \leq u_{i}^{\max } \\ u_{i}^{\min } & u_{i}<u_{i}^{\min }\end{array}\right.$ denotes the input saturation function. For $x \in \Re^{n},|x(t)|_{1}=\sum_{i=1}^{n}\left|x_{i}(t)\right|$ denotes the 1-norm.

\section{Problem Formulation}

Consider the IMC structure as shown in Figure 1. Define

$$
y^{\prime}(t)=(P * \hat{u})(t)=\int_{0}^{t} P(t-\tau) \hat{u}(\tau) d \tau
$$

Thus $y^{\prime}$ corresponds to the output of the constrained system. Because of the saturation constraints, $y^{\prime}(t)$ necessarily differs from $y(t)$, the output for the unconstrained system. In general, we would like to keep $y^{\prime}$ as close to $y$ as possible. Mathematically, we want to solve the following optimization problem instantaneously at each time $t$.

$$
\min _{\hat{i}}\left|(f * y)(t)-\left(f * y^{\prime}\right)(t)\right|_{1}=\min _{\hat{u}}|(f P Q * e)(t)-(f P * \hat{u})(t)|_{1}
$$

where $f$ is a filter such that $f P$ is biproper. If $P$ is strictly proper, then $\hat{u}$ does not affect $y^{\prime}$ instantaneously and the minimization is meaningless. Since our ultimate goal is to minimize $\left|y(t)-y^{\prime}(t)\right|_{1}, f$ must be diagonal in order not to introduce any change in the output direction.

The minimization is carried out continuously for $t \geq 0$. It is important to realize that this instantaneous minimization differs from the minimization over a horizon. For the conventional IMC structure displayed in Figure 1, $\hat{u}(t)=\operatorname{sat}(u(t))=\operatorname{sat}\left(\int_{0}^{t} Q e(\tau) d \tau\right)$ is completely determined for any given $e(t)$. Thus, in general, the conventional IMC implementation does not solve optimization problem (2) which optimizes the 


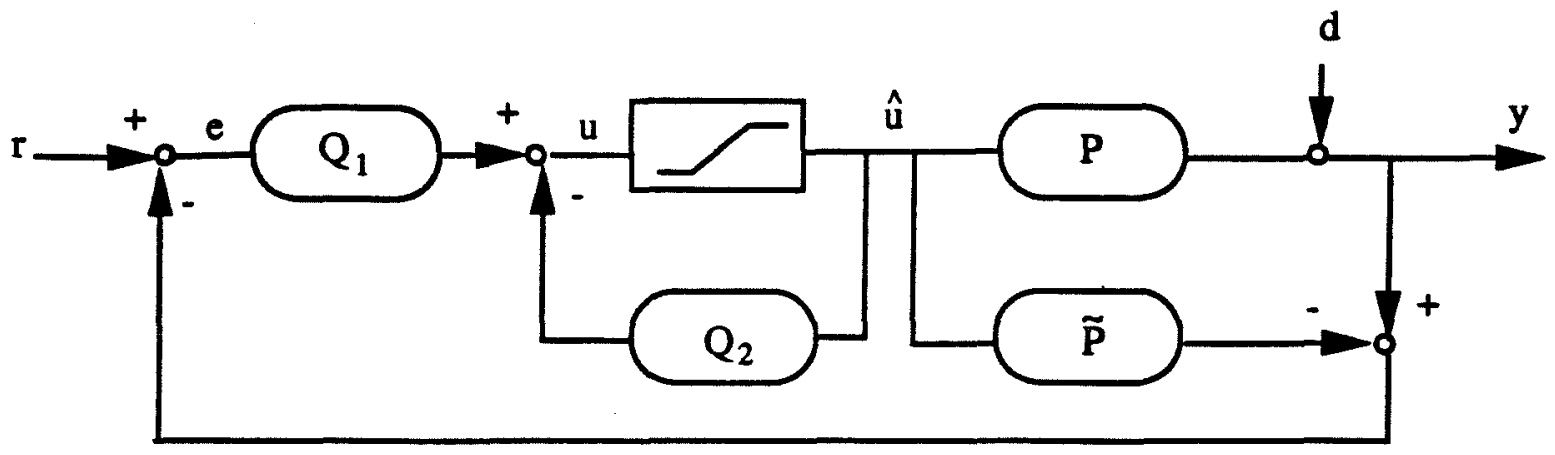

Figure 2: Modified IMC Structure

performance for the constrained system. In the next section, we will show that a modified IMC structure actually solves the optimization problem (2) instantaneously.

\section{Anti-windup Design}

\subsection{IMC Structure}

Figure 2 shows the modified IMC structure where $Q=\left(I+Q_{2}\right)^{-1} Q_{1}$. Assume that $Q$ is biproper. ${ }^{1}$ We have

$$
u(s)=Q_{1} e(s)-Q_{2} \hat{u}(s)=Q_{1} e(s)-\left(Q_{1} Q^{-1}-I\right) \hat{u}(s)
$$

In the time domain,

$$
u(t)-\hat{u}(t)=\left(Q_{1} * e\right)(t)-\left(Q_{1} Q^{-1} * \hat{u}\right)(t)
$$

The following lemma states how $f$ should be chosen such that the modified IMC structure shown in Figure 2 solves the optimization problem (2).

Lemma 1 Suppose that $Q$ is biproper and that $P=\tilde{P}$. If $\left.f P\right|_{s=\infty}$ is a diagonal nonsingular matrix with finite elements and $Q_{1}=f P Q$, then $\hat{u}(t)$ resulting from the modified IMC implementation (Figure 2 ) is the solution of optimization problem (2). Furthermore, if $g=D f$ where $D$ is a diagonal constant matrix, then the closed-loop responses with $f$ and $g$ are identical.

Proof: $Q_{1}=f P Q \Rightarrow u(t)-\hat{u}(t)=(f P Q * e)(t)-(f P * \hat{u})(t)=(f * y)(t)-\left(f * y^{\prime}\right)(t) \equiv y_{f}(t)-y_{f}^{\prime}(t)$. We have

$$
u_{i}(t)-\hat{u}_{i}(t)=y_{f_{i}}(t)-y_{f_{i}}^{\prime}(t), \quad i=1,2, \cdots, n .
$$

Since $\left.f P\right|_{e=\infty}$ is diagonal, $\hat{u}_{j}, j \neq i$, do not affect $y_{f_{i}}^{\prime}$ instantaneously. Equations (5) can be solved independently for each $\hat{u}_{i}(t)$. Consider the first input, i.e. $i=1$. When no saturation occurs at $t=t_{1}$, $\hat{u}_{1}\left(t_{1}\right)=u_{1}\left(t_{1}\right)=\operatorname{sat}\left(u_{1}\left(t_{1}\right)\right)$ and $\left|y_{f_{1}}\left(t_{1}\right)-y_{f_{1}}^{\prime}\left(t_{1}\right)\right|=0$ is minimized. Suppose that saturation occurs at $t=t_{2}$, i.e. $u_{1}\left(t_{2}\right)>u_{1}^{\max }$ or $u_{1}\left(t_{2}\right)<u_{1}^{\min }$, we want to show that $\dot{u}_{1}\left(t_{2}\right)=\operatorname{sat}\left(u_{1}\left(t_{2}\right)\right)$ also minimizes $\left|y_{f_{1}}\left(t_{2}\right)-y_{f_{1}}^{\prime}\left(t_{2}\right)\right|$. Since $\hat{u}_{1}\left(t_{2}\right)$ affects $y_{f_{1}}^{\prime}\left(t_{2}\right)$ linearly and $\hat{u}_{j}\left(t_{2}\right), j=2,3, \cdots, n$, do not affect $y_{f_{1}}^{\prime}\left(t_{2}\right)$, $\left|y_{f_{1}}\left(t_{2}\right)-y_{f_{1}}^{\prime}\left(t_{2}\right)\right|$ is a convex function of $\hat{u}_{1}\left(t_{2}\right)$ only. If $\hat{u}_{1}\left(t_{2}\right)=u_{1}\left(t_{2}\right)$ for which $\left|y_{f_{1}}\left(t_{2}\right)-y_{f_{1}}^{\prime}\left(t_{2}\right)\right|=0$ is not feasible, i.e. $u_{1}\left(t_{2}\right)>u_{1}^{\text {mas }}$

\footnotetext{
${ }^{1} Q$ is biproper if both $Q$ and $Q^{-1}$ are proper.

${ }^{2}$ Here zero initial condition is assumed. This is without loss of generality since $Q$ is stable and nonzero initial conditions can be incorporated into $e(t)$.
} 
or $u_{1}\left(t_{2}\right)<u_{1}^{m i n}$, then the optimal solution which minimizes $\left|y_{f_{1}}\left(t_{2}\right)-y_{f_{1}}^{\prime}\left(t_{2}\right)\right|$ must occur at the boundary, i.e. $\hat{u}_{1}\left(t_{2}\right)=\operatorname{sat}\left(u_{1}\left(t_{2}\right)\right)$. Therefore, choosing $\hat{u}_{1}(t)=\operatorname{sat}\left(u_{1}(t)\right)$ minimizes $\left|y_{f_{1}}(t)-y_{f_{1}}^{\prime}(t)\right|$ for each $t \geq 0$. Since $\left|y_{f_{i}}(t)-y_{f_{i}}^{\prime}(t)\right|$ is minimized for each $i,\left|y_{f}(t)-y_{f}^{\prime}(t)\right|_{\ell}$ is minimized.

If $g=D f$, Equations (5) become

$$
u_{i}(t)-\hat{u}_{i}(t)=D_{i i}\left[y_{f_{i}}(t)-y_{f_{i}}^{\prime}(t)\right], \quad i=1,2, \cdots, n .
$$

where $D=\operatorname{diag}\left\{D_{11}, \cdots, D_{n n}\right\}$. Before saturation occurs, the system is unconstrained and $\hat{u}(t)=u(t)$ does not depend on $D$. Assume that system saturates for input 1 at $t=t_{1}$, then $\hat{u}_{1}\left(t_{1}\right)=u_{1}^{\text {mas }}$ or $\hat{u}_{1}\left(t_{1}\right)=u_{1}^{\text {min }}$. As long as the right hand side of Equation (5) does not become zero for $i=1$, input 1 stays saturated and $\hat{u}_{1}(t)$ is constant during this period. Input 1 becomes unsaturated only if the right hand side of Equation (5) becomes zero for $i=1$ which is not a function of $D_{11}$. Therefore, the system comes out of the saturation at the same time regardless of what $D_{11}$ is. Similar arguments can be used when more than one input saturates. Therefore, the closed-loop responses for $f$ and $g$ are identical.

Remark 1 If $\left.f P\right|_{o=\infty}$ is not diagonal, then $y_{f_{i}}^{\prime}(t)$ may also be affected by $\hat{u}_{j}(t), j \neq i$, instantaneously. The convexity argument would not work since $\left|y_{f_{i}}(t)-y_{f_{i}}^{\prime}(t)\right|$ is also affected by $\hat{u}_{j}(t), j \neq i$.

Remark $2 f$ must be diagonal in order not to introduce any change in the output direction. However, $f$ for which $\left.f P\right|_{0=\infty}$ is diagonal may not be diagonal. To get around this problem, we can design a diagonal $f$ for $\tilde{P}$ such that $\left.f \tilde{P}\right|_{s=\infty}$ is diagonal. $\tilde{P}$ can be chosen arbitrarily close to $P . Q_{2}$ must be strictly proper to be implementable. This can be achieved by choosing $f$ appropriately.

Remark $3 Q$ is usually minimum phase and always stable. If $Q$ is minimum phase and $Q_{1}$ non-minimum phase, then $\left(I+Q_{2}\right)^{-1}$ must be unstable. Therefore, $Q_{1}$ must be minimum phase and stable to guarantee internal stability of the closed-loop system. $f$ must be chosen such that $f P Q$ is both minimum phase and stable.

Remark 4 For the modified IMC structure, the input is kept saturated for an optimal amount of time until $\left|y_{f}(t)-y_{f}^{\prime}(t)\right|$ becomes zero. Thus, in general, the performance is greatly improved when $f$ is appropriately chosen.

Different controller factorizations can be obtained by choosing $f$ differently. We discuss two special cases here.

Case 1: $f=P^{-1}$. The optimization problem (2) becomes $\min _{\tilde{u}}|u(t)-\hat{u}(t)|_{1}$. The solution corresponds to the conventional IMC structure which "chops off" the control input resulting in performance deterioration. However, stability of the closed-loop system is guaranteed.

Case 2: $f$ is such that $Q_{1}$ is a constant matrix. The optimization becomes $\min _{i} \mid Q_{1}\left[e(t)-e^{\prime}(t) \|_{1}\right.$, where $e^{\prime}(t)=\left(Q^{-1} * \hat{u}\right)(t)$. This factorization corresponds to the Model State Feedback proposed in [4] for SISO systems. The performance in this case is greatly improved, but stability of the closed-loop system is not guaranteed. If the dynamics of $P Q$ are slow, however, minimizing the weighted controller input error $\left(e(t)-e^{\prime}(t)\right)$ may not be a good way to optimize the nonlinear performance. After the system comes out of the nonlinear region, the controller takes no action to compensate for the effect of the error, $e(t)-e^{\prime}(t)$, introduced during the saturation. 


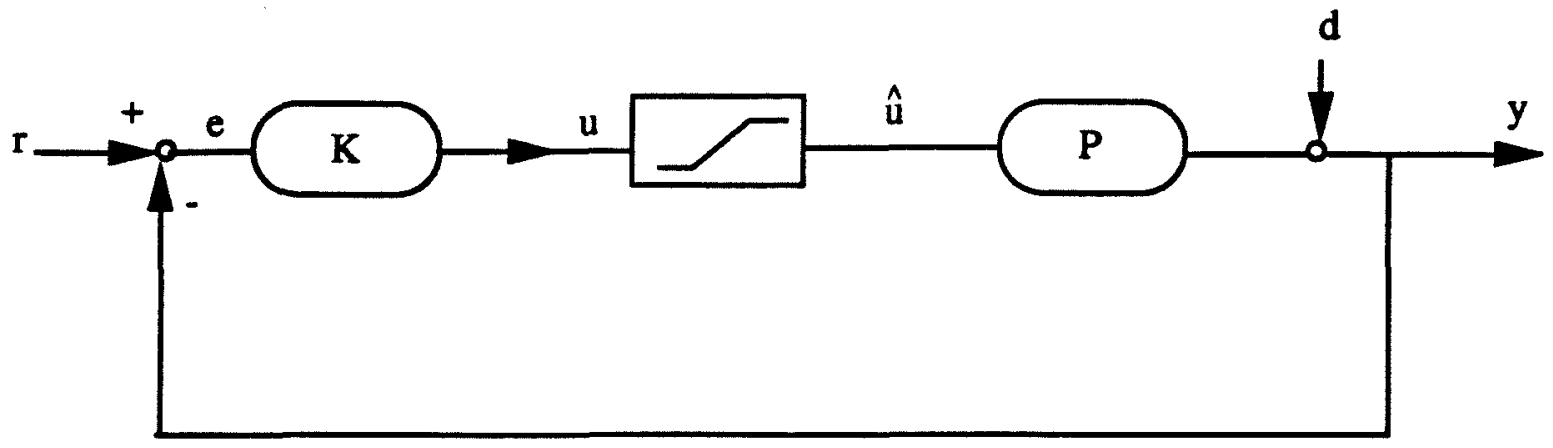

Figure 3: Classical Feedback Structure

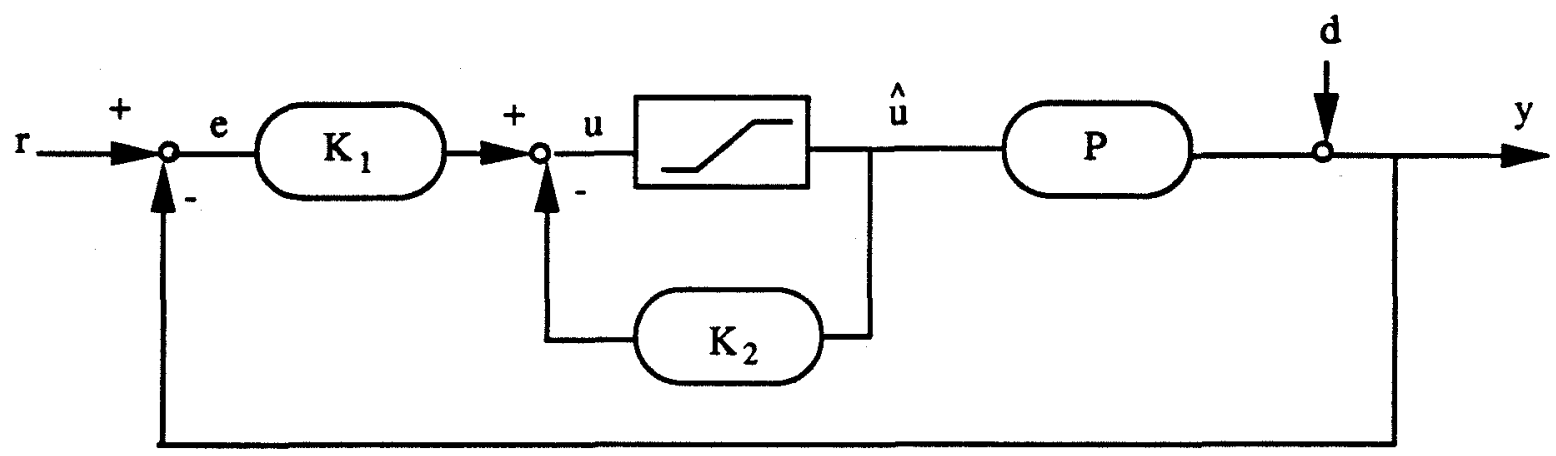

Figure 4: Classical Feedback Structure with Anti-windup

In Case $1 f$ was chosen to guarantee stability while $f$ was chosen to enhance performance in Case 2. Therefore, $f$ can generally be tuned to trade off performance and stability of the constrained system. It should be pointed out that $f$ in Case 2 was not an extreme choice.

\subsection{Classical Feedback Structure}

For stable systems, the IMC structure shown in Figure 1 and the classical feedback structure shown in Figure 3 are equivalent. The results for the modified IMC structure can be extended directly to the classical feedback structure to obtain the anti-windup structure shown in Figure 4. The controllers $K_{1}$ and $K_{2}$ are defined as follows:

$$
\begin{aligned}
K_{1} & =Q_{1} \\
K_{2}(s) & =Q_{2}-Q_{1} \tilde{P}
\end{aligned}
$$

Hanus [8, 9] suggested the following

$$
\begin{aligned}
K_{1} & =K^{\prime}(\infty) \\
K_{2}(s) & =K_{1} K^{-1}(s)-I
\end{aligned}
$$

where $K=Q(I-\tilde{P} Q)^{-1}$. This factorization corresponds to $f=K_{1} Q^{-1} P^{-1}$. Therefore, Banus's conditioning technique minimizes $\mid K_{1}\left[e(t)-e^{\prime}(t)\right] \|_{1}$ and represents the MIMO extension of the Model State Feedback. 


\section{Examples}

In this section, several examples are shown to demonstrate the effectiveness of the proposed method.

Example 1 Consider the following plant:

$$
P(s)=\frac{2}{100 s+1}
$$

The IMC controller designed for a step input is

$$
Q(s)=\frac{100 s+1}{2(20 s+1)}
$$

Case 1. Choosing $f=2.5(20 s+1)$ gives

$$
\begin{aligned}
& Q_{1}=2.5 \\
& Q_{2}=\frac{4}{100 s+1}
\end{aligned}
$$

Case 2. Choosing $f=50(s+1)$ gives

$$
\begin{aligned}
& Q_{1}=\frac{50(s+1)}{20 s+1} \\
& Q_{2}=\frac{99}{100 s+1}
\end{aligned}
$$

Here $f(\infty)$ was chosen such that $Q_{2}$ is strictly proper. The input is constrained between the saturation limits \pm 1 . The responses to a unit step disturbance with the conventional IMC and the modified IMC implementations are shown in Figures 5 and 6 along with the unconstrained responses. The figures illustrate the sluggishness of performance of the conventional IMC implementation when the closed loop dynamics are much faster than those of the open loop. For the conventional IMC implementation, the saturation effectively "chops off" the control input resulting in performance deterioration. The modified IMC implementation keeps the control signal saturated for an optimum length of time as discussed in Section 3 resulting in improved performance. $f$ in Case 1 corresponds to minimizing $\left|e(t)-e^{\prime}(t)\right|$ while $f$ in Case 2 corresponds approximately to minimizing $\left|y(t)-y^{\prime}(t)\right|$. The control input in Case 2 stays saturated until $y(t) \approx y^{\prime}(t)$ while the control input in Case 1 stays saturated until $e(t)=e^{\prime}(t)$. In Case 1 , the difference between $y(t)$ and $y^{\prime}(t)$ resulting from the difference between $e(t)$ and $e^{\prime}(t)$ during the saturation is not compensated as can be seen in Figure 5.

Example 2 This example is taken from [6] where the conventional anti-windup method did not result in a stable closed loop system. The plant is a fourth order lag-lead butterworth:

$$
P=0.2\left(\frac{s^{2}+2 \xi_{1} \omega_{1} s+\omega_{1}^{2}}{s^{2}+2 \xi_{1} \omega_{2} s+\omega_{2}^{2}}\right)\left(\frac{s^{2}+2 \xi_{2} \omega_{1} s+\omega_{1}^{2}}{s^{2}+2 \xi_{2} \omega_{2} s+\omega_{2}^{2}}\right)
$$

where $\omega_{1}=0.2115, \omega_{3}=0.0473, \xi_{1}=0.3827$ and $\xi_{2}=0.9239$.

The IMC controller is

$$
Q=\frac{s+1}{(16 s+1) P}
$$

Choosing $f=\frac{3(16 \iota+1)}{16(0+1)}$ gives

$$
\begin{aligned}
Q_{1} & =\frac{5}{16} \\
Q_{2}(s) & =\frac{5}{16 Q(s)}-1
\end{aligned}
$$




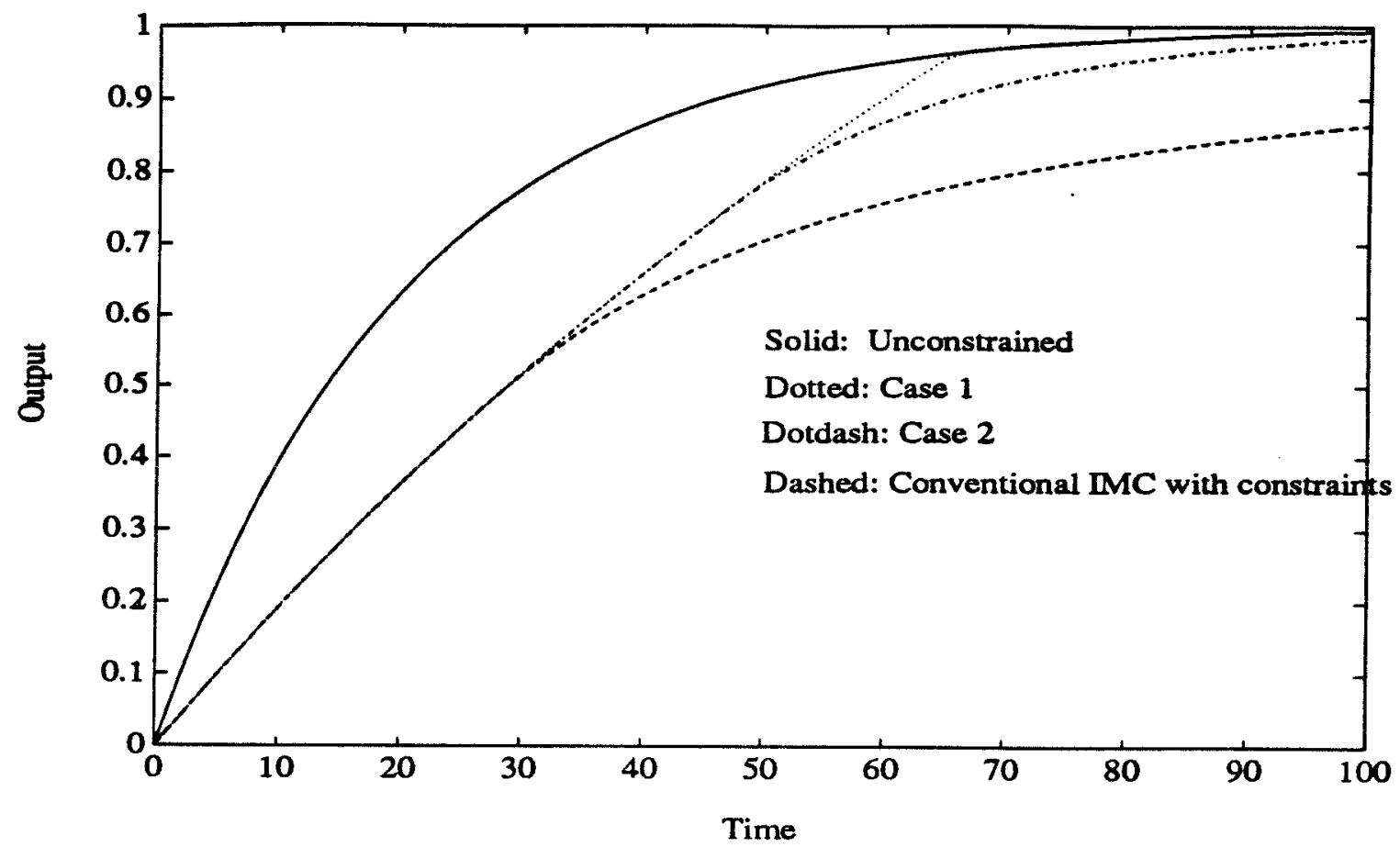

Figure 5: Example 1 - Plant Output Responses

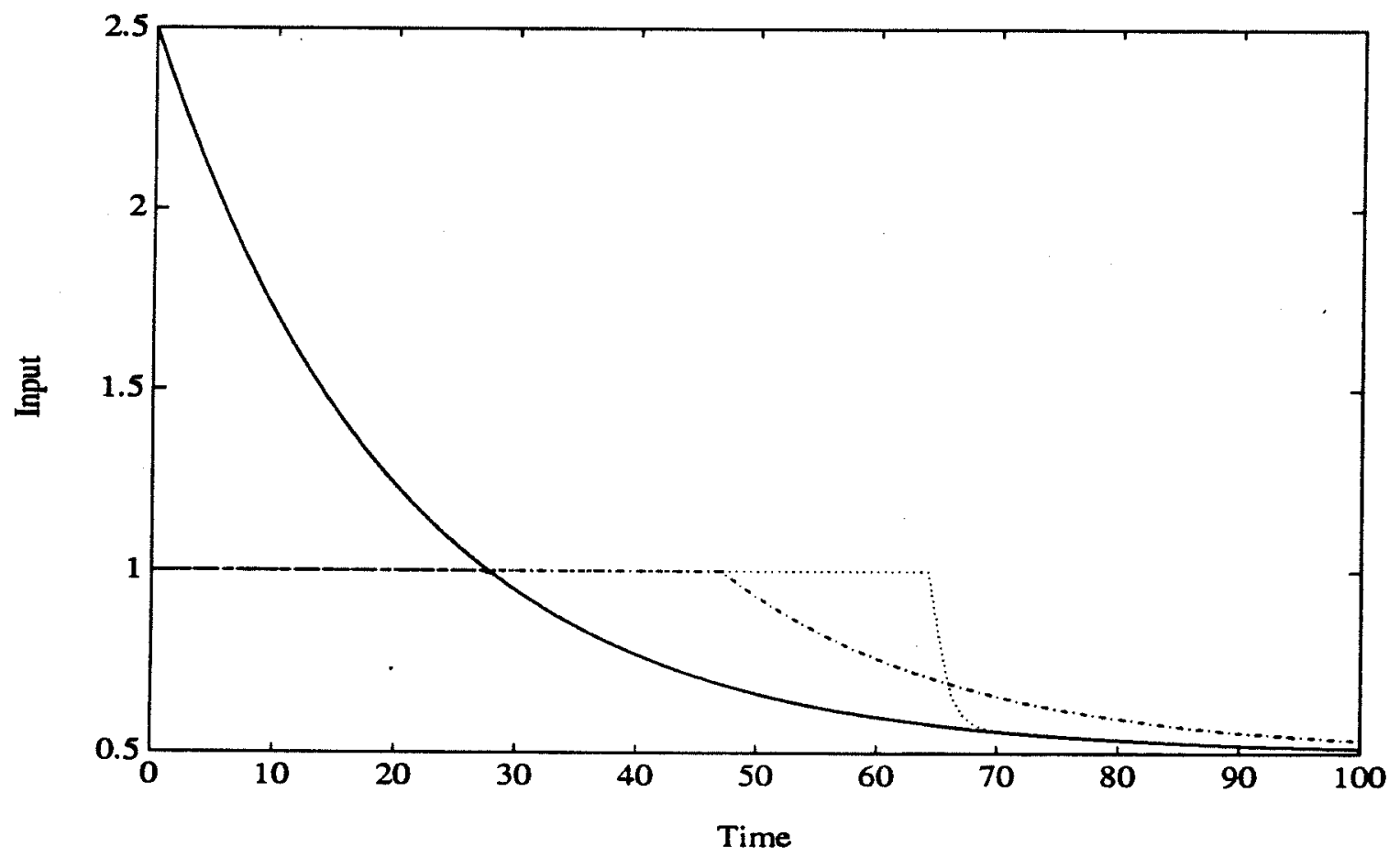

Figure 6: Example 1 - Coutroller Output Responses 


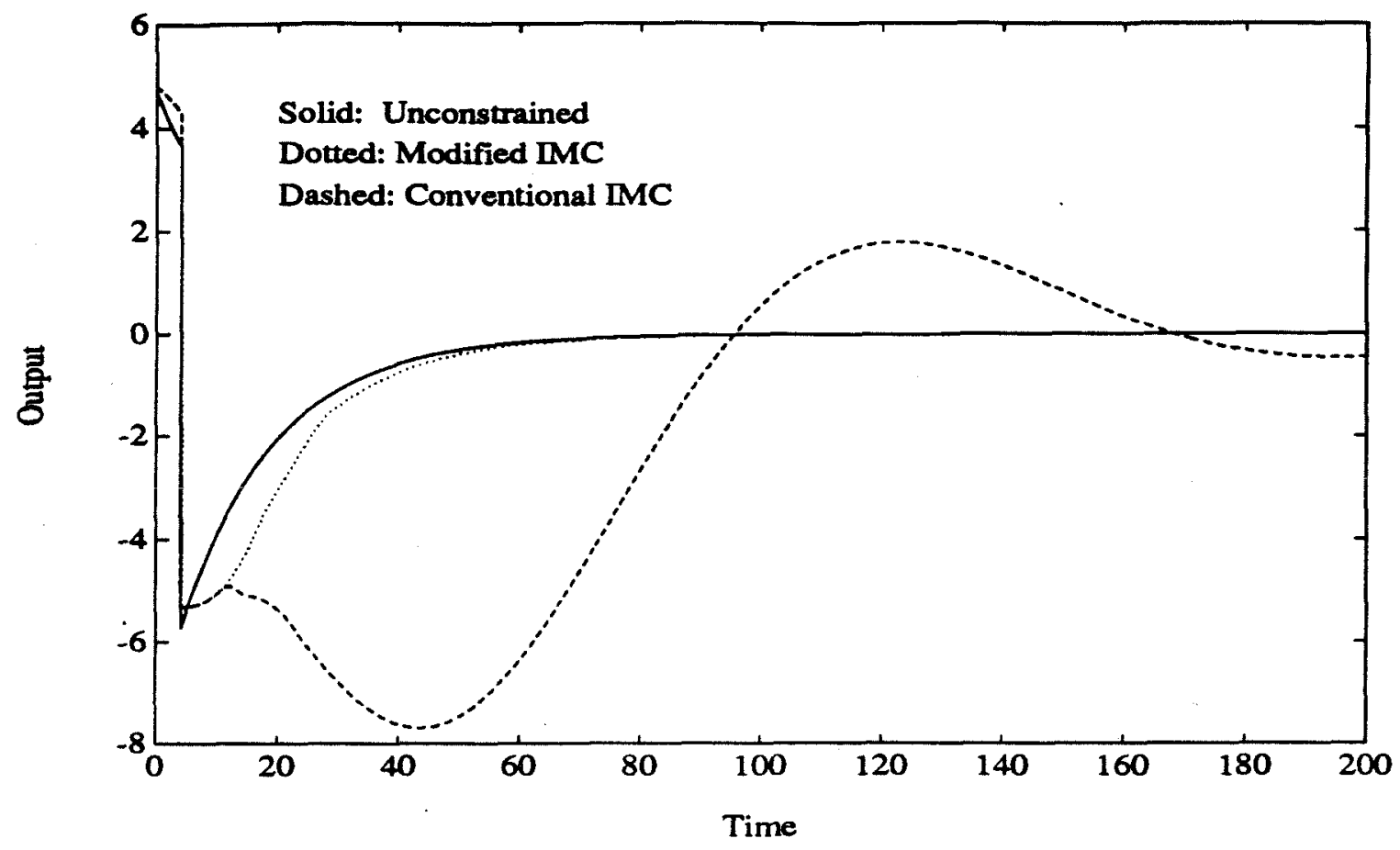

Figure 7: Example 2 - Plant Output Responses

The input is constrained between the saturation limits \pm 1 . Figure 7 shows the responses for a disturbance input with step of magnitude of 5 at time $t=0$ and a switch to -5 at $t=4$. The performance improvement over the conventional IMC implementation is significant. Furthermore, the off-axis criterion [5] can be used to show that the closed-loop system is globally asymptotically stable.

Example 3 Consider the following plant:

$$
P(s)=\frac{10}{100 s+1}\left[\begin{array}{cc}
4 & -5 \\
-3 & 4
\end{array}\right]
$$

Both inputs are constrained between the saturation limits \pm 1 . A setpoint change of $\left[\begin{array}{ll}0.63 & 0.79\end{array}\right]^{T}$ is applied. The IMC controller designed for a step input is

$$
Q(s)=\frac{100 s+1}{10(20 s+1)}\left[\begin{array}{ll}
4 & 5 \\
3 & 4
\end{array}\right]
$$

Two values of $f$, one diagonal and one non-diagonal, are chosen to see how $f$ (diagonal or not diagonal) affects the closed-loop performance.

\section{Case 1.}

$$
\begin{aligned}
f & =10(s+1)\left[\begin{array}{ll}
4 & 5 \\
3 & 4
\end{array}\right] \\
Q_{1} & =f P Q \\
Q_{2} & =f P-I
\end{aligned}
$$




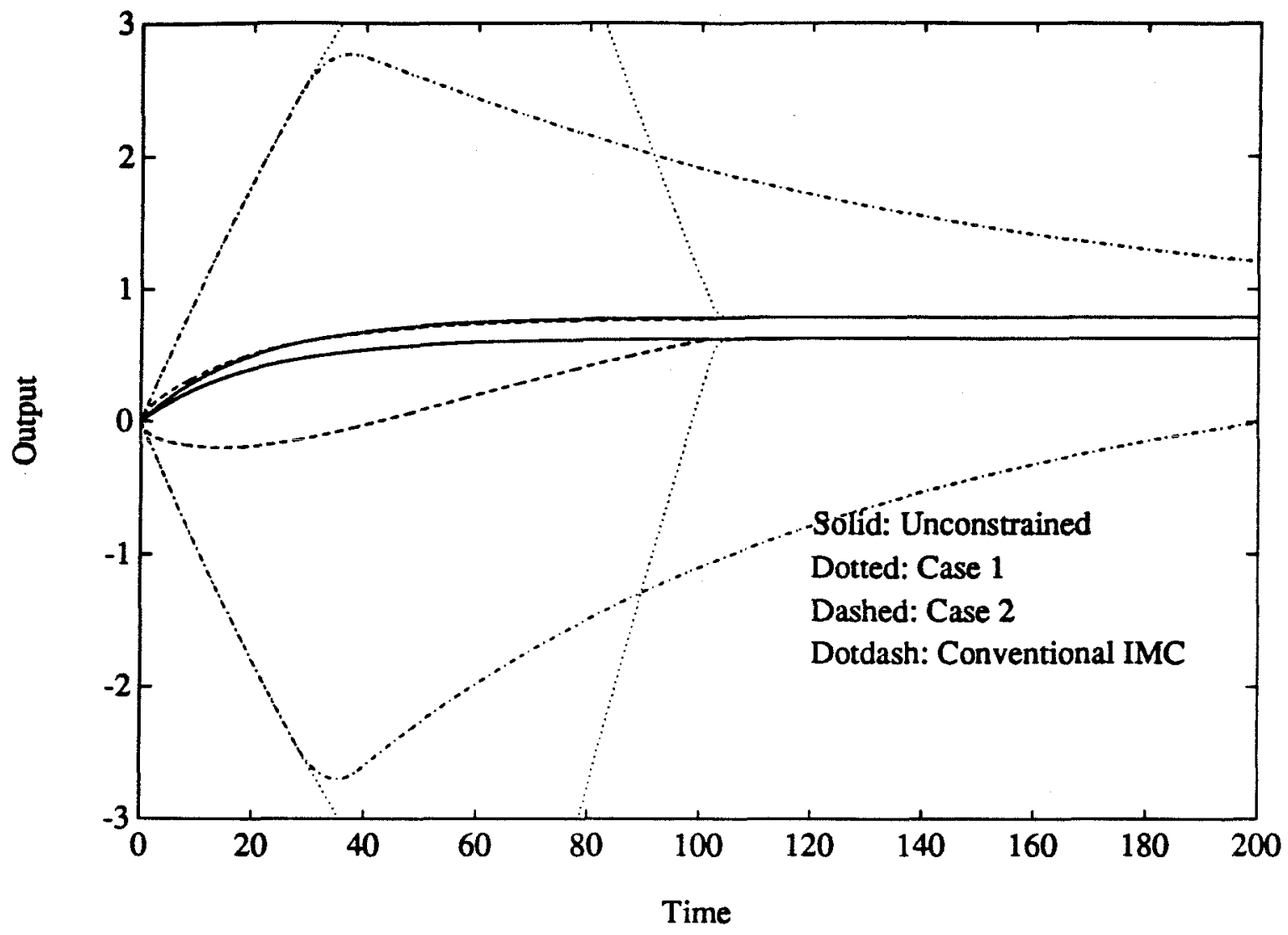

Figure 8: Example 3 - Plant Output Responses

Case 2.

$$
\begin{aligned}
f & =2.5(s+1) I \\
\tilde{P} & =\frac{10}{100 s+1}\left[\begin{array}{cc}
4 & \frac{-5}{0.1 s+1} \\
\frac{-3}{0.1 s+1} & 4
\end{array}\right] \\
Q_{1} & =f \tilde{P} Q \\
Q_{2} & =f \tilde{P}-I
\end{aligned}
$$

The responses for both cases and the conventional IMC implementation are shown in Figure 8. As we can see, choosing $f$ to be a diagonal nonsingular matrix is crucial to obtain good nonlinear performance. In Case 1 , some direction of the outputs is minimized.

\section{Conclusions}

We have proposed an anti-windup scheme which optimizes the error between the outputs of the system generated by the constrained and unconstrained inputs, respectively. The method generalizes the Model State Feedback for SISO systems proposed in [4] and Hanus's conditioning technique. In particular, the 
Model State Feedback corresponds to choosing $f$ such that $Q_{1}$ is constant; Hanus's conditioning technique corresponds to choosing $f$ such that $Q_{1}=K(\infty)$. Furthermore, the derivation presented here elucidates the objectives behind the different methods and clearly shows the consequences of the various parameter choices. As shown by Example 3, the performance for $Q_{1}=K(\infty)$ for MIMO systems may suffer when $K(\infty)$ is not diagonal. Examples illustrate that this scheme provides graceful degradation of performance. The attractive features of the scheme are its simplicity and effectiveness. The filter $f$ can be tuned to trade off performance and stability of the co nstrained system.

\section{Acknowledgement}

Partial support from the National Science Foundation is gratefully acknowledged.

\section{References}

[1] K. J. Åström and L. Rundqwist. Integrator windup and how to avoid it. In Proceedings of the 1989 American Control Conference, 1693-1698, 1989.

[2] K. J. Åström and B. Wittenmark. Computer Controlled Systems Theory and Design. Prentice-Hall, Inc., Englewood Cliffs, N.J., 1984.

[3] P. J. Campo and M. Morari. Robust control of processes subject to saturation nonlinearities. Computers $\mathcal{E}$ Chemical Engineering, 14(4/5):343-358, 1990.

[4] E. Coulibaly, S. Maiti and C. Brosilow. Internal Model Predictive Control. AIChE Annual Meeting, Miami, FL, 1992.

[5] Y. S. Cho, K. S. Narendra. An Off-axis Circle Criterion for the Stability of Feedback Systems with a Monotonic Nonlinearity. IEEE Transactions on Automatic Control, AC-13, 1968.

[6] J. C. Doyle, R. S. Smith and D. F. Enns. Control of plants with input saturation nonlinearities. In Proceedings of the 1987 American Control Conference, 1034-1039, 1987.

[7] H. A. Fertik and C. W. Ross. Direct digital control algorithms with anti-windup feature. ISA Thans., 6(4):317-328, 1967.

[8] R. Hanus and M. Kinnaert. Control of constrained multivariable systems using the conditioning technique. In Proceedings of the 1989 American Control Conference, pages 1711-1718, 1989.

[9] R. Hanus, M. Kinnaert and J. L. Henrotte. Conditioning technique, a general anti-windup and bumpless transfer method. Automatica, 23(6):729-739, 1987.

[10] M. Morari and E. Zafiriou. Robust Process Control. Prentice-Hall, Inc., Englewood Cliffs, N.J., 1989.

[11] K. S. Walgama and J. Sternby. Inherent observer property in a class of anti-windup compensators. International Journal of Control, 52(3):705-724, 1990. 